\title{
Neurocognitive disorders: what are the prioritized caregiver needs? A consensus obtained by the Delphi method
}

Teddy Novais ${ }^{1,2,3^{*}}$, Christelle Mouchoux ${ }^{2,3,4}$, Michel Kossovsky ${ }^{5}$ Lucie Winterstein ${ }^{6}$, Floriane Delphin-Combe ${ }^{6}$, Pierre Krolak-Salmon ${ }^{3,4,6}$ and V. Dauphinot ${ }^{6}$

\begin{abstract}
Background: The symptoms related to neurocognitive disorders (NCD) may lead to caregiver burden increase. Involving caregivers in research may be an effective way of improving the practicalities and relevance of interventions. The aim of this study was to gather opinion and gain consensus on the caregivers 'priorities, using a Delphi method and including aspects of needs in pharmaceutical dimension.

Methods: Observational study using a modified Delphi method. This study was conducted in the Clinical and Research Memory Center of the University Hospital of Lyon (France), between September 2015 and January 2016. The expert panel was composed of 68 informal caregivers of people with subjective cognitive decline or NCD living at home.

Results: Caregivers assigned a very high importance to the dimension "information needs about their relative's disease", i.e. information on the disease, the treatment and the research; and to "coping skills", i.e. skills related to emotional support, communication, relationship evolution with the relative and skills to cope with behavioural crisis, behavioural and cognitive disorders. The aspect "coping with behavioural disorders" received a high selection rate (83\%).

Conclusions: The main needs selected can be used to design relevant interventions and give guidance to policy to support caregivers. To meet caregiver's needs, interventions should focus on information about disease and treatment and psychoeducational interventions.
\end{abstract}

Keywords: Caregivers, Neurocognitive disorders, Delphi method, Needs assessment, Training/education

\section{Background}

Cognitive impairment, functional autonomy loss and behavioural disorders caused by neurocognitive disorders (NCD) as Alzheimer's Disease and Related Diseases (ADRD) may lead to caregiver burden increase $[1,2]$. The informal caregiver, i.e. not professional, is often a spouse or a child, providing assistance with daily living activities, managing the relative's behavioural symptoms, coordinating supportive services, facilitating healthcare visits and making financial and healthcare decisions [3]. They play a central role in the care of people with NCD given caregiver informal care account for more than half

\footnotetext{
* Correspondence: teddy.novais@chu-lyon.fr

${ }^{1}$ EA-7425 HESPER, Health Services and Performance Research, University Lyon, F-69003 Lyon, France

${ }^{2}$ Pharmaceutical Unit, Charpennes Hospital, Hospices Civils de Lyon, F-69100 Lyon, France

Full list of author information is available at the end of the article
}

of societal costs [4] However, the burden of care may have physical, psychological, emotional, social and financial consequences on caregiver and increase caregiver frailty may cause early patient institutionalization [5, 6]. In addition, previous studies have shown a large number of unmet needs were associated with a higher burden and an increase in caregiver strain and depressive symptoms [3, 7-9]. In NCD, the number of unmet needs is high while the levels of services utilization are low [10]. Is there a discrepancy between the services and support proposed to caregivers and the services that they need?

Involving informal caregivers to understand their needs from their own perspectives may be an effective way of improving the practicalities, acceptability and relevance of services and support [11].

Several studies using quantitative or qualitative approaches have evaluated the caregivers' needs in terms

(c) The Author(s). 2018 Open Access This article is distributed under the terms of the Creative Commons Attribution 4.0 International License (http://creativecommons.org/licenses/by/4.0/), which permits unrestricted use, distribution, and reproduction in any medium, provided you give appropriate credit to the original author(s) and the source, provide a link to the Creative Commons license, and indicate if changes were made. The Creative Commons Public Domain Dedication waiver (http://creativecommons.org/publicdomain/zero/1.0/) applies to the data made available in this article, unless otherwise stated. 
of information, skills, support and services in medical and psychosocial dimensions $[12,13]$. However, it appears that the needs assessment instruments used in these studies did not explore all dimensions of needs. This is the case for the needs in pharmaceutical dimension while people with NCD and their caregivers represent populations with higher risk of developing drug-related problems due to aging, comorbidities associated with polypharmacy and caregiver neglect of their own health care [14-19]. Previous studies have identified an amount of needs in informal caregivers but no study has hierarchized their main needs in medical, pharmaceutical and psychosocial dimensions. Identifying the main caregivers'needs and hierarchizing them would allow to design targeted and appropriate interventions to meet the needs of the greatest number. The aim of this study was to gather opinion and gain consensus on the caregivers 'priorities, using a Delphi method and including aspects of needs in pharmaceutical dimension. The intended outcome was to identify the main common needs among informal caregivers of people with NCD.

\section{Methods}

\section{Study design}

A two round modified Delphi process was used to generate consensus amongst a panel of informal caregivers. The Delphi method was chosen because this method allows to define a consensus in panel of experts on a specific topic from a large number of predefined items with a technique for the indirect confrontation of opinions [20]. This method also allows to hierarchize the main items retained round after round by the experts. The Delphi process uses a series of questionnaires to collect information from participants in a number of rounds. In the case of modified Delphi, the starting point is a pre-derived list of questions established by analysts [21]. A decision was made to restrict the Delphi process to two rounds before inviting experts to participate, since the initial questionnaire was based upon a careful review of available literature. It was predicted that two rounds would be enough to reach adequate consensus and would minimise the burden and the difficulty of the process for the caregivers of people with NCD.

\section{Setting and participants}

Informal caregivers, considered as experts of this Delphi survey, were recruited at the Clinical and Research Memory Center of the University Hospital of Lyon (Hospices Civils de Lyon, France), between the September 1, 2015 and January 30, 2016. Inclusion criteria were: informal caregivers of people living at home with NCD and agreeing to participate to the Delphi survey [22]. The informal caregiver was defined as a nonprofessional person living with their relative or providing support to their relative.

\section{Detailed Delphi study scheme \\ Development of the survey questionnaire}

The first step was to develop the survey questionnaire for the first round. The aspects of needs in the survey were based on a systematic review whose purpose was to review the methodologies used to identify the needs, the existing needs assessment instruments and the main topics of needs explored among caregivers of patients with mild cognitive impairment to dementia [12]. The selection criteria were: studies using quantitative, qualitative and mixed method to assess the caregiver's needs in terms of information, coping skills, support and service. Several aspects of needs related to pharmaceutical care have been added to the questionnaire. The first draft of the questionnaire was developed by the first author and was independently reviewed by analysts specialized in the care of ADRD patients to establish content validity. The group of analysts included 2 geriatricians, a neuro-geriatrician, 2 neuropsychologists, 2 pharmacists, a social worker and a methodologist. This allowed the identification of 46 aspects of needs into 3 dimensions: (i) 19 aspects in the dimension of information needs (INF), (ii) 18 aspects in the dimension of skills needs (SKI) and (iii) 9 aspects in the dimension of support needs (SUP). Fifteen of the 46 aspects of needs involved the pharmaceutical field, including information about caregiver's and patient's drugs and medication management.

\section{Round 1 method}

First, an information letter explaining the study and the procedure for the first round was given to the caregivers after a memory consultation of their relatives. Second, the first questionnaire was distributed to caregivers who agreed to participate.

The first round consisted of rating the pre-selected aspects of needs. Caregivers were asked to rate the importance of each of these 46 aspects on a nine-point scale with three anchors: 1 "not important"; 5 "of moderate importance"; 9 "extremely important". Respondents were also informed that they could add other aspects of needs.

The second round was based on a second questionnaire drawn up from the responses obtained at the first round, using two criteria to determine the retained aspects: they had to be both "consensual" and "important", i.e. to have obtained $75 \%$ of the answers in one of the three parts of the scale [1-9] and a median score $\geq 8$. This threshold has been shown to favour high reliability [23].

\section{Round 2 method}

The second questionnaire of the Delphi process was sent to the home of the same panel of experts for the second round. In each round, stamped envelopes were provided to the informal caregivers to send back the questionnaire. 
The second round aimed to select the main aspects of needs according to the same panel of experts, i.e. aspect of needs selected by $50 \%$ and over of caregivers (representing the majority of caregivers). Amongst the proposed aspects in the second questionnaire, caregivers were asked to select the ten aspects that they considered to be "the most important" and to rank them in order of importance from 1 to 10 , with 10 being the most important and 1 the least important. The rank given by the caregiver for each aspect of the second questionnaire was equated to a weight of importance (e.g. the rank 10 equals a weight of 10).

The second round analysis was based on the number of experts who selected each aspect of needs and the total weight of importance (sum of the weights granted by caregivers for each aspect). The more an aspect of needs was selected by the experts and more its total weight was important, the more this aspect was important for the caregivers.

In the second round, questions about the caregivers 'preferences regarding the implementation of identified needs in information, skills and support were also added in another questionnaire. The preferences in terms of intervention timing (i.e. on the demand, one shot at the diagnosis or several times all along the disease) and means (i.e. leaflet, internet, individual or collective session, hotline) were investigated.

\section{Data collection}

Data collected at the inclusion included: sociodemographic characteristics (age, gender, educational level), relationship with the relative, the length of caregiving, the caregiver burden using the short version of Zarit Burden Interview (ZBI) and patient medical data: diagnosis, global cognitive function using the Mini Mental State Examination (MMSE), behaviour using the Neuropsychiatric Inventory (NPI), the functional autonomy level using the Instrumental Activities of Daily Living (IADL). The short version of ZBI score ranged from 0 (no burden) to 7 (higher burden) [5, 24]. The French version of MMSE was previously validated for the detection of cognitive impairment using the DSM-III criteria [25]. The IADL assessed 8 instrumental activities and the score ranging from 0 (dependent) to 8 (independent) [26]. The NPI evaluates 10 behavioral domains and 2 neurovegetative troubles [27]. A higher overall NPI score (maximum 144) indicates more severe behavioral disorders. All data were entered in an electronic database to perform the analyses.

\section{Data analysis}

Relative with NCD and caregivers characteristics were described at baseline with means \pm standard deviations (SD) or frequencies (percentages). The participation rate to the second round of the survey was reported and characteristics of caregivers who completed the two round and those who achieved only the first round were compared. Normally distributed data were compared using the student's t test and paired categorical variables using Chi-square test. For the round 1, the results were described using means \pm SD and median of the rating assigned on the scale to each aspect of needs. The response rates in the three parts of the scale [1-9] was also described for each aspect of needs. For the round 2, the frequency of selection and the weight of each aspect of needs from the second questionnaire were detailed. The weight of an aspect of needs corresponds to the sum of the rank (10 to 1 ) granted by the caregivers. Data were analysed using the Excel software (Microsoft Office Excel 2010) and SPSS (Statistical Package for the Social Sciences) version 21.0 for Windows (SPSS Inc., Chicago, Illinois, USA).

\section{Ethical consideration}

The observational study protocol has been reviewed and approved by a local ethics committee the 8/7/2015 (Comité de Protection des Personnes Sud-Est IV, references L15-119).

\section{Results \\ Panel of experts}

Between September 2015 and January 2016, sixty eight caregivers of people with NCD participated in the first round of the survey. Fifty eight caregivers (85\%) participated to the second round. There were no significant differences between the groups that participated in only round 1 and completed in both. The characteristics of the caregivers and their relatives were summarized in Table 1.

\section{Round 1}

The mean score of importance of the 46 aspects was $6.93 \pm 2.49$, varying according to the dimensions of needs (Table 2). The SUP dimension was the least important, with a mean score of $4.98 \pm 2.94$. In this dimension, the caregivers attributed the lowest scores to the pharmaceutical support. They attributed a mean score of $7.33 \pm$ 2.07 for the INF dimension and $7.46 \pm 2.17$ for the SKI dimension. In these dimensions, the highest scores were attributed to the needs of information about their relative's disease and providing affective support to their relative. Twenty aspects of needs met the first decision-rule made to draw up the second questionnaire. No aspect of needs in support has been selected for the second round.

Several aspects of needs were assessed as important but not consensual (dissensus): 9 areas of needs with a median score $\geq 8$ were assessed as "extremely important" by 61 to $74 \%$ of the caregivers in the round 1 . This notably concerned skills to cope with feeling related to 
Table 1 Experts i.e. informal caregivers' characteristics and people with NCD characteristics

\begin{tabular}{|c|c|}
\hline & $n=68$ \\
\hline \multicolumn{2}{|l|}{ Gender - n (\%) } \\
\hline female & $46(67.5 \%)$ \\
\hline \multicolumn{2}{|l|}{ Relationship with their relative- $\mathrm{n}(\%)$} \\
\hline spouses & $43(63.0 \%)$ \\
\hline children & $20(29.5 \%)$ \\
\hline other & $5(7.5 \%)$ \\
\hline Age - mean \pm SD & $69.1 \pm 12.0$ \\
\hline age of spouses - mean $\pm S D$ & $75.3 \pm 8.5$ \\
\hline age of children - mean $\pm S D$ & $56.0 \pm 7.1$ \\
\hline age $\geq 65-n(\%)$ & $42(62 \%)$ \\
\hline \multicolumn{2}{|l|}{ Educational level - n (\%) } \\
\hline nil & $5(7.5 \%)$ \\
\hline primary & $9(13.0 \%)$ \\
\hline secondary & $33(48.5 \%)$ \\
\hline tertiary & $21(31.0 \%)$ \\
\hline \multicolumn{2}{|l|}{ Cohabitation with their relative $-\mathrm{n}(\%)$} \\
\hline yes & $49(72.0 \%)$ \\
\hline \multicolumn{2}{|l|}{ Length of caregiving - $\mathrm{n}(\%)$} \\
\hline$<1$ year & $12(17.5 \%)$ \\
\hline $1-5$ year(s) & $48(70.5)$ \\
\hline$>5$ years & $8(12.0 \%)$ \\
\hline Caregiver Burden (short ZBI) - mean \pm SD & $3.7 \pm 1.9$ \\
\hline \multicolumn{2}{|l|}{ People with NCD characteristics } \\
\hline Age - mean $\pm S D$ & $80.0 \pm 8.0$ \\
\hline \multicolumn{2}{|l|}{ Gender - n (\%) } \\
\hline Female & $35(51.5 \%)$ \\
\hline \multicolumn{2}{|l|}{ Measurements - mean \pm SD } \\
\hline Mini-Mental State Examination & $17.4 \pm 6.2$ \\
\hline Neuropsychiatric Inventory & $21.8 \pm 14.8$ \\
\hline Instrumental Activities of Daily Living & $3.0 \pm 2.8$ \\
\hline Disability Assessment of Dementia DAD-16 & $5.3 \pm 5.8$ \\
\hline \multicolumn{2}{|l|}{ Diagnosis stage - n (\%) } \\
\hline Subjective cognitive decline & $4(6.0 \%)$ \\
\hline Minor neurocognitive disorders & $15(22.0 \%)$ \\
\hline Major neurocognitive disorders & $49(72.0 \%)$ \\
\hline \multicolumn{2}{|l|}{ Diagnosis etiology - n (\%) } \\
\hline Alzheimer's Disease & $38(56.0 \%)$ \\
\hline $\begin{array}{l}\text { Alzheimer's Disease with cerebrovascular } \\
\text { disease }\end{array}$ & $12(17.5 \%)$ \\
\hline Lewy body disease & $4(6.0 \%)$ \\
\hline Cerebrovascular disease & $5(7.0 \%)$ \\
\hline Other neurocognitive disorders & $9(13.5 \%)$ \\
\hline
\end{tabular}

ZBI Zarit Burden Index caring, to communicate on the relatives' disease, and caregiving with their entourage; as well as information needs about non-pharmacological treatment and nursing care at home. No other aspect of needs was added by the respondents for the second round after the questionnaires analysis.

\section{Round 2}

The questionnaire of the second round was composed of 20 aspects of needs evaluated as "consensual" and "important" by the expert panel. Ten needs aspects (top ten list) were selected by $50 \%$ and over caregivers (50 to $83 \%$ ). Amongst the ten most selected needs aspects, four pertained to the dimension "information needs about the relative's disease", i.e. information on the disease, the treatment and the research; and six to "coping skills", i.e. skills related to emotional support, communication, relationship evolution with the relative and skills to cope with behavioural crisis, behavioural and cognitive disorders (Table 3). The aspect "coping with behavioural disorders" received a high selection rate (83\%). However, seven aspects of needs among the ten remaining aspects were also reported to be of importance for more than one third of the caregivers.

\section{Focus on pharmaceutical care needs}

Amongst the fifteen aspects of needs in pharmaceutical dimension in the round 1 , three aspects were ranked as important and consensual by the caregivers such as research (therapies and disease), information about patient's drugs for the cognitive disease and information about patient's drugs for comorbidities. However, two others aspects were ranked as important but not consensual (dissensus): adjustment of sedative drug dosage and the management of the patient's drug. The aspects of needs about informal caregivers' medication were the lowest ranked. In the round 2 , two aspects of needs in pharmaceutical dimension were included in the top ten list (selected by $50 \%$ and over of caregivers): research and information about patient's drugs for the cognitive disease (rank 8 and 10 respectively).

\section{Preferences about information delivery and skill acquisition}

Table 4 summarizes the caregivers' preferences. For the information delivery and the skill acquisition, the majority of caregivers said that they would prefer multiple individual interviews with an appropriate health professional all along the disease progression of their relatives.

\section{Discussion}

The Delphi findings present an informal caregiver consensus on their priority needs in the field of NCD.

Previous studies using other methodologies have evaluated the caregiver's needs in NCD. A systematic review 
Table 2 First round - rating of the 46 needs aspects $(n=68)$

\begin{tabular}{|c|c|c|c|c|c|c|}
\hline \multirow[b]{2}{*}{ Needs aspects } & \multirow[b]{2}{*}{$\mathrm{DIM}^{\mathrm{a}}$} & \multirow[b]{2}{*}{ Median } & \multirow[b]{2}{*}{ Mean (SD) } & \multicolumn{3}{|c|}{ Distribution of the ratings along the scale (\%) } \\
\hline & & & & $1-3$ & $4-6$ & $7-9$ \\
\hline Information on the neurocognitive disease and its progression ${ }^{b}$ & INF-P & 9 & $8.39(1.10)$ & 0 & 7 & 93 \\
\hline Providing emotional and affective support to PWNCD ${ }^{b}$ & SKI-C & 9 & $8.32(1.32)$ & 2 & 6 & 92 \\
\hline Information on cognitive disorders ${ }^{b}$ & INF-P & 9 & $8.23(1.13)$ & 0 & 9 & 91 \\
\hline The diagnostic process of the neurocognitive disease ${ }^{b}$ & INF-P & 9 & $8.19(1.24)$ & 0 & 11 & 89 \\
\hline Communication between caregivers and PWNCD ${ }^{b}$ & SKI-C & 9 & $8.17(1.41)$ & 0 & 14 & 86 \\
\hline Management of the PWNCD pain ${ }^{b}$ & SKI-C & 9 & $8.17(1.35)$ & 2 & 6 & 92 \\
\hline Coping with cognitive disorders ${ }^{b}$ & SKI-C & 9 & $8.11(1.39)$ & 0 & 15 & 85 \\
\hline Coping with behavioral crisis at home ${ }^{b}$ & SKI-C & 9 & $8.09(1.66)$ & 2 & 12 & 86 \\
\hline Coping with behavioral disorders ${ }^{\mathrm{b}}$ & SKI-C & 9 & $8.01(1.49)$ & 1 & 12 & 87 \\
\hline The research (disease and therapies) ${ }^{b}$ & INF-P & 9 & $8.01(1.34)$ & 0 & 16 & 84 \\
\hline $\begin{array}{l}\text { Roles of the health professionals involved in the PWNCD } \\
\text { care pathway }\end{array}$ & INF-P & 9 & $7.96(1.39)$ & 0 & 15 & 85 \\
\hline Information on behavioral disorders ${ }^{b}$ & INF-P & 9 & $7.94(1.74)$ & 5 & 10 & 85 \\
\hline $\begin{array}{l}\text { Information on the drugs for the neurocognitive disease } \\
\text { (efficacy, tolerance and utilization) }{ }^{b}\end{array}$ & INF-P & 9 & $7.84(1.69)$ & 4 & 14 & 82 \\
\hline Undertaking the steps for the services utilization ${ }^{b}$ & SKI-S & 9 & $7.50(2.35)$ & 9 & 13 & 78 \\
\hline Finding available services closer to home ${ }^{b}$ & SKI-S & 9 & $7.49(2.31)$ & 7 & 15 & 78 \\
\hline $\begin{array}{l}\text { Learning to adjust the sedative drug dose based on the } \\
\text { patient's condition (e.g. sedation, agitation) }\end{array}$ & SKI-M & 8.5 & $6.97(2.7)$ & 14 & 19 & 67 \\
\hline $\begin{array}{l}\text { Coping with the relationship evolution between caregivers } \\
\text { and PWNCD }\end{array}$ & SKI-C & 8 & $7.83(1.40)$ & 0 & 20 & 80 \\
\hline Stimulating/appropriates activities for PWNCD at home ${ }^{b}$ & $\mathrm{SKI}-\mathrm{C}$ & 8 & $7.79(1.59)$ & 1 & 19 & 80 \\
\hline Basic care to PWNCD ${ }^{b}$ & SKI-C & 8 & $7.74(1.67)$ & 2 & 15 & 83 \\
\hline $\begin{array}{l}\text { Information on the PWNCD drugs for comorbidities (efficacy, } \\
\text { tolerance and utilization) }\end{array}$ & INF-P & 8 & $7.56(1.66)$ & 3 & 21 & 76 \\
\hline $\begin{array}{l}\text { Coping with feeling related to caring (e.g, frustration, } \\
\text { culpability, stress) }\end{array}$ & SKI-C & 8 & $7.53(1.79)$ & 3 & 23 & 74 \\
\hline Financial issues for the $A D L^{b}$ & INF-S & 8 & $7.5(2.19)$ & 8 & 13 & 79 \\
\hline Information on non-pharmacological treatment for PWNCD & INF-P & 8 & $7.45(1.59)$ & 0 & 30 & 70 \\
\hline $\begin{array}{l}\text { Communicating on the PWNCD disease and the caregiver } \\
\text { role with the entourage }\end{array}$ & SKI-C & 8 & $7.44(1.85)$ & 3 & 26 & 71 \\
\hline Information on nursing care at home & INF-S & 8 & $7.24(2.41)$ & 9 & 17 & 74 \\
\hline Legal issues & INF-S & 8 & $6.98(2.24)$ & 7 & 26 & 67 \\
\hline Information on respite, day care & INF-S & 8 & $6.90(2.65)$ & 13 & 17 & 70 \\
\hline Information on institutionalization and long-term care unit & INF-S & 8 & $6.90(2.50)$ & 11 & 25 & 64 \\
\hline Being able to manage the PWNCD drugs & SKI-M & 8 & $6.55(2.87)$ & 20 & 20 & 61 \\
\hline Information on the consequences of caregiving (caregiver health) & INF-C & 7 & $7.09(1.97)$ & 5 & 29 & 66 \\
\hline Learning to seek and accept help from the entourage & SKI-C & 7 & $6.92(2.01)$ & 3 & 35 & 62 \\
\hline Information on associations and foundations & INF-S & 7 & $6.601 .93)$ & 5 & 38 & 57 \\
\hline Information on environmental safety & INF-S & 7 & $6.342 .37)$ & 12 & 33 & 55 \\
\hline Coping with difficulties to administer the PWNCD drugs & SKI-M & 7 & $6.22(2.82)$ & 20 & 25 & 55 \\
\hline $\begin{array}{l}\text { Review of the PWNCD drug prescribing by a pharmacist } \\
\text { (optimization of the therapeutics) }\end{array}$ & SUP-P & 7 & $6.01(2.68)$ & 18 & 31 & 51 \\
\hline Being able to manage their drugs (caregiver's drugs) & SKI-M & 7 & $5.40(3.33)$ & 35 & 14 & 51 \\
\hline Annual medical check-up for caregiver by a GP & SUP & 6.5 & $6.23(2.70)$ & 17 & 33 & 50 \\
\hline Information on drugs for caregiver's psychological disorders & INF-C & 6 & $6.25(3.35)$ & 10 & 45 & 45 \\
\hline
\end{tabular}


Table 2 First round - rating of the 46 needs aspects ( $n=68)$ (Continued)

\begin{tabular}{|c|c|c|c|c|c|c|}
\hline \multirow[b]{2}{*}{ Needs aspects } & \multirow[b]{2}{*}{$\mathrm{DIM}^{\mathrm{a}}$} & \multirow[b]{2}{*}{ Median } & \multirow[b]{2}{*}{ Mean (SD) } & \multicolumn{3}{|c|}{ Distribution of the ratings along the scale (\%) } \\
\hline & & & & $1-3$ & $4-6$ & $7-9$ \\
\hline $\begin{array}{l}\text { Information on the caregiver's drugs (efficacy, tolerance and } \\
\text { utilization) }\end{array}$ & INF-C & 6 & $5.98(2.58)$ & 16 & 42 & 42 \\
\hline Support by a social worker & SUP & 5 & $5.67(2.92)$ & 24 & 36 & 40 \\
\hline Obtaining a form with timing of PWNCD drug administration & SUP-P & 5 & $5.62(2.82)$ & 20 & 39 & 41 \\
\hline $\begin{array}{l}\text { Identification and resolution of difficulties associated with the } \\
\text { PWNCD drug management }\end{array}$ & SUP-P & 5 & $5.44(2.77)$ & 22 & 39 & 29 \\
\hline Emotional support for the caregiver by a psychologist & SUP & 5 & $4.76(2.56)$ & 29 & 44 & 27 \\
\hline $\begin{array}{l}\text { Review of the caregiver drug prescribing by a pharmacist } \\
\text { (optimization of the therapeutics) }\end{array}$ & SUP-P & 2.5 & $3.62(2.81)$ & 53 & 28 & 19 \\
\hline $\begin{array}{l}\text { Identification and resolution of the difficulties associated with the } \\
\text { caregiver's drug management }\end{array}$ & SUP-P & 2.5 & $3.52(2.70)$ & 53 & 28 & 19 \\
\hline Obtaining a form with timing of caregiver drug administration & SUP-P & 2 & $3.42(2.90)$ & 58 & 23 & 19 \\
\hline
\end{tabular}

$A D L$ activities of the daily living, GP general practitioner, $P W N C D$ people with a neurocognitve dirsorder

${ }^{a}$ Dimension of need. INF-P information needs about patient (people with NCD), INF-C information needs about caregiving, INF-S information needs about services, SKI-C coping skills needs, SKI-M Skills needs about medication, SKI-S skills needs about services, SUP support needs for the caregiver, SUP-P pharmaceutical support ${ }^{\mathrm{b}}$ Aspect of need selected for the second round

Table 3 Second round - selecting of the 20 needs aspects $(n=58)$

\begin{tabular}{|c|c|c|c|c|c|}
\hline \multirow[t]{2}{*}{ Aspect of needs } & \multirow[t]{2}{*}{$\mathrm{DIM}^{\mathrm{a}}$} & Selection & Percent & Weight & Rank \\
\hline & & \multicolumn{4}{|l|}{ Number of experts } \\
\hline Coping with behavioral disorders & SKI-C & 48 & 83 & 307 & 1 \\
\hline Coping with behavioral crisis & $\mathrm{SKI}-\mathrm{C}$ & 41 & 71 & 280 & 2 \\
\hline Information on the neurocognitive disease and its progression & INF-P & 37 & 64 & 240 & 3 \\
\hline Information on behavioral disorders & INF-P & 38 & 66 & 219 & 4 \\
\hline Providing emotional and affective support to PWNCD & SKI-C & 35 & 60 & 214 & 5 \\
\hline Communication between caregivers and PWNCD & SKI-C & 28 & 48 & 177 & 6 \\
\hline Coping with cognitive disorders & SKI-C & 28 & 48 & 175 & 7 \\
\hline The research (disease and therapies) & INF-P & 37 & 64 & 170 & 8 \\
\hline $\begin{array}{l}\text { Coping with the relationship evolution between caregivers } \\
\text { and PWNCD }\end{array}$ & $\mathrm{SKI}-\mathrm{C}$ & 30 & 52 & 152 & 9 \\
\hline $\begin{array}{l}\text { Information on the drugs for the neurocognitive disease } \\
\text { (efficacy, tolerance and utilization) }\end{array}$ & INF-P & 29 & 50 & 147 & 10 \\
\hline Information on cognitive disorders & INF-P & 23 & 40 & 146 & - \\
\hline Stimulating/appropriates activities for PWNCD at home & SKI-C & 25 & 43 & 133 & - \\
\hline Management of the PWNCD pain & $\mathrm{SKI}-\mathrm{C}$ & 21 & 36 & 128 & \\
\hline $\begin{array}{l}\text { Roles of the health professionals involved in the PWNCD } \\
\text { care pathway }\end{array}$ & INF-P & 22 & 38 & 111 & - \\
\hline Finding available services closer to home & SKI-S & 28 & 48 & 100 & - \\
\hline Undertaking the steps for the services utilization & SKI-S & 26 & 45 & 100 & - \\
\hline Financial issues for the $A D L$ & INF-S & 23 & 40 & 94 & - \\
\hline The diagnostic process of the neurocognitive disease & INF-P & 18 & 31 & 92 & - \\
\hline Basic care to PWNCD & $\mathrm{SKI}-\mathrm{C}$ & 15 & 26 & 85 & - \\
\hline $\begin{array}{l}\text { Information on the PWNCD drugs for comorbidities } \\
\text { (efficacy, tolerance and utilization) }\end{array}$ & INF-P & 11 & 19 & 41 & - \\
\hline
\end{tabular}

$A D L$ activities of the daily living, $P W N C D$ people with a neurocognitve dirsorder

${ }^{a}$ Dimension of need. INF-P information needs about patient (people with NCD); INF-S information needs about services, SKI-C coping skills needs, SKI-S skills needs about services 
Table 4 Caregivers' preferences about information delivery and skills acquisition features

\begin{tabular}{llll}
\hline Means of information delivery & $\%$ & Means of skills acquisition & Timing \\
\hline Timing & 72 & Several times all along the disease \\
Several times all along the disease & 18 & One shot, at the diagnosis of the disease \\
One shot, at the diagnosis of the disease & 10 & On demand \\
On demand & 0 & Other \\
Other & & Means \\
Means & 53 & Personal interview \\
Personal interview & 19 & Collective session \\
Internet site & 13 & Hotline \\
Leaflet or video & 15 & Other \\
Collective workshop & 0 & 30 \\
Other & & 21 \\
\hline
\end{tabular}

of 12 qualitative studies has identified two aspects of needs: management of older people with dementia and caregivers' personal needs [13]. The first aspect included similar needs to the present study: information and disease knowledge, activities of daily living support, behavioral and psychological symptoms of dementia support and, formal/informal care support. The second aspect included caregivers' physical and psychological health and management of caregivers' own lives. Wackerbarth et al. have used quantitative method through self-administered needs assessment surveys [28]. Caregivers of individuals with ADRD were asked to rate information and support needs in terms of importance. The most important information needs involved the health plan coverage (financial issue), the means to find the best care and information about diagnosis and therapeutics, especially current treatment options, risks and benefits and treatments for behaviors. The most important support needs identified by caregivers were mainly for the relative as providing emotional support and understand the relative's feelings. Rather than support for themselves, caregivers rated support for their relatives as most important [28]. Similarly, in the present Delphi study, informal caregivers have given low rank to the domains of needs about their own concerns such as emotional support for the caregiver and annual medical check-up by general practitioner for the caregiver. Caregivers often appear to ignore their own personal and health needs to concentrate on providing the best care possible to the ill person [29-32]. This is more noticeable with medication-related needs, as caregivers did not perceive needs for their own medication management, at least not as much as for their relatives. Older people themselves, informal caregivers are also exposed to polypharmacy with a higher risk of developing drug-related problems. Thorpe et al. have shown that 33\% of patients living with dementia and $39 \%$ of their caregivers were taking at least one potentially inappropriate medication [18]. These aspects of pharmaceutical needs mainly met the needs of health professionals while informal caregivers did not perceive possible benefits.

The Delphi process is rarely used in non-professional subjects, especially in informal caregivers to establish a consensus. Bond et al. developed a set of guidelines on how family and non-professional helpers may assist an older person who is developing neurocognitive impairment, or has dementia or delirium [31]. They used two expert panels: health professional panel and caregiver advocate panel (people who were members of the national Alzheimer's organization or a caregiver organization and held a leadership position within an advocacy organi zation). Family or informal caregivers were not included in the building of these guidelines. Another study using the Delphi process in dementia was conducted with professionals and informal caregivers of people living with dementia to identify the main resilience factors i.e. personal strategies helping to face difficulties related to the disease [32]. The items patients' behavioral problems and feeling competent as a caregiver were selected as essential resilience features. Caregivers also emphasized the importance of social supports, the quality of the relationship with their relative, while professionals considered coping skills, and a good quality of life of caregivers most relevant. These studies indicated that priorities of caregiver advocates/informal caregivers and professionals differed to some extent [11, 32].

Dickinson et al. and Gilhooly et al. showed in their Meta-analyzes that the most effective psychosocial interventions for caregivers were multicomponent and psychoeducational interventions [33, 34]. In the meta-analysis of Sörensen et al., psychoeducational interventions were defined as interventions that "involve a structured program geared toward providing information about the care receiver's disease process and about resources and services and training caregivers to respond effectively to diseaserelated problems, such as memory and behavior problems in dementia patients" [35]. In this present study, ten 
common caregiver's needs are identified allowing to design a common core of educational and psychoeducational interventions. To meet their needs, these interventions should be focused on (1) information about the disease, the behavioral disorders, the medication and research; (2) coping skills regarding behavioral and cognitive disorders, behavioral crisis at home, relationship changes; and (3) skills to communicate and to provide affective support to their relatives. In addition, specific needs, i.e. important but not consensual needs (dissensus), have been also highlighted in the first round.

A multicomponent intervention integrating personalized pharmaceutical care to a multidisciplinary psychosocial program for caregivers of people with ADRD (PHARMAID study protocol) was designed and is being evaluated [36]. This collaborative program aims to optimize the medication management of the patient with ADRD and their caregiver, to provide appropriate information and to develop coping skills based on collective and individual interventions. The finding of this study will assess the effectiveness of this collaborative approach.

\section{Strengths and limitations}

To our knowledge, this is the first study reporting prioritized informal caregivers' needs in NCD by using a Delphi process. A main strength of the present study is the involvement of caregivers in a Delphi survey to be experts of their situation and to identify their proper needs. Using a consensus of people with real-life experience allows highlighting information and suggestions that may be relevant and useful to caregivers dealing with cognitive and behavioral disorders. The use of a ranking process in the second round enables caregivers to provide a clear prioritization of their needs. Another advantage of the Delphi methodology is the possibility to obtain anonymously the views of a diverse group of persons, preventing the influence of dominant individuals $[32,37]$. The present study has been conducted in a single-centre specialized in the care of people with cognitive complaints in France and with a mostly urban population of caregivers. Despite the high number of recruited experts as compared to other Delphi studies $[38,39]$, the present study identified needs that may not be internationally extendable. Indeed, a large variability in care structures and organizations in the medico-social field is observed across countries, as well as discrepancy in health care accessibility between urban and rural caregivers. The first round questionnaire of the Delphi method was developed form a systematic review of quantitative and qualitative studies and proposed a wide range of needs. However, even if the group of analysts who validated this questionnaire had a global vision on health and social-care provision, it did not include all professionals involved in the care of people with NCD (eg. nurses, occupational therapists). Moreover, the questionnaire of the first round was distributed after the consultation with the specialist. The rating could be affected by the service and content of memory consultation (e.g. if the caregiver felt that memory consultation could not provide adequate information on the disease of cognitive disorder, the subject may rate that it was very important to provide information on cognitive disorder). A measure of the caregiver satisfaction or dis-satisfaction about the memory consultation could have prevented this limitation. Finally, caregivers' needs may be subject to factors, such as relationship to their relative, disease stage, caregiving length and others. The sample size of caregivers did not allow to achieve subgroup analysis.

\section{Conclusion}

The main needs selected from informal caregivers perspectives can be used to design relevant intervention studies and give guidance to policy to support caregivers of people with NCD more effectively and better tailored to their needs. To meet the informal caregiver's needs and to reduce their burden, interventions focused on disease and treatment information and psychoeducational interventions should be prioritized. In order to provide personalized support to informal caregivers, this study should be replicated internationally in rural and urban caregivers and individual needs should be assessed ahead of the educational process.

\section{Abbreviations}

ADRD: Alzheimer's disease and Related Disorders; INF: Information needs; NCD: Neurocognitive Disorders; SD: Standard Deviations; SKI: Skills needs; SUP: Support needs

\section{Acknowledgements}

We are indebted to all the French experts who agreed to participate in the Delphi study. We thank also other analysts of this study: Romain Bachelet, Nathalie Jomard, and Marie-Hélène Michel.

\section{Funding}

None.

\section{Availability of data and materials}

The datasets generated during the current study are available from the corresponding author upon reasonable request.

\section{Authors' contributions}

The survey questionnaire was designed by a group of analysts: TN (PharmD, PhD candidate), VD (PhD), CM (PharmD, PhD), MK (MD), PKS (MD, PhD), NJ (MD), FDC, RB, and MHM. The establishment of the panel of experts was carried out by LW and TN. The data analysis was performed by TN and VD. The manuscript was written by TN. The critical revision of the manuscript was made by all authors. All authors read and approved the final manuscript.

\section{Ethics approval and consent to participate}

The observational study protocol has been reviewed and approved by a local ethics committee the 8/7/2015 (Comité de Protection des Personnes Sud-Est IV, references L15-119). To be included in this observational study, the ethics committee approved that participants give verbal consent after being informed about the study. The verbal consent of the caregiver was documented in the patient medical record. 


\section{Consent for publication}

Not applicable.

\section{Competing interests}

The authors declare that they have no competing interests.

\section{Publisher's Note}

Springer Nature remains neutral with regard to jurisdictional claims in published maps and institutional affiliations.

\section{Author details}

'EA-7425 HESPER, Health Services and Performance Research, University Lyon, F-69003 Lyon, France. ${ }^{2}$ Pharmaceutical Unit, Charpennes Hospital, Hospices Civils de Lyon, F-69100 Lyon, France. ${ }^{3}$ University Lyon 1, F-69000 Lyon, France. ${ }^{4}$ INSERM U1028, CNRS UMR5292, Lyon Neuroscience Research Center, Brain Dynamics and Cognition Team, F-69000 Lyon, France. ${ }^{5}$ Department of Internal medicine, Rehabilitation and Geriatrics, University Hospitals of Geneva and University of Geneva, Geneva, Switzerland. ${ }^{6}$ Clinical and Research Memory Centre of Lyon (CMRR), Charpennes Hospital, Hospices Civils de Lyon, F-69100 Lyon, France.

Received: 4 January 2018 Accepted: 17 December 2018 Published online: 29 December 2018

\section{References}

1. Vellas B, Hausner L, Frölich L, Cantet C, Gardette V, Reynish E, et al. Progression of Alzheimer disease in Europe: data from the European ICTUS study. Curr Alzheimer Res. 2012;9(8):902-12.

2. Dauphinot V, Ravier A, Novais T, Delphin-Combe F, Mouchoux C, KrolakSalmon P. Risk factors of caregiver burden evolution, for patients with subjective cognitive decline or neurocognitive disorders: a longitudinal analysis. J Am Med Dir Assoc. 2016;17(11):1037-43.

3. Black BS, Johnston D, Rabins PV, Morrison A, Lyketsos C, Samus QM. Unmet needs of community-residing persons with dementia and their informal caregivers: findings from the maximizing independence at home study. J Am Geriatr Soc. 2013;61(12):2087-95.

4. Rapp T, Andrieu S, Chartier F, Deberdt W, Reed C, Belger M, et al. Resource Use and Cost of Alzheimer's Disease in France: 18-Month Results from the GERAS Observational Study. Value Health. 2017; article in Press.

5. Zarit SH, Reever KE, Bach-Peterson J. Relatives of the impaired elderly: correlates of feelings of burden. The Gerontologist. 1980;20(6):649-55.

6. Yaffe K, Fox P, Newcomer R, Sands L, Lindquist K, Dane K, et al. Patient and caregiver characteristics and nursing home placement in patients with dementia. JAMA. 2002;287(16):2090-7.

7. Rosa E, Lussignoli G, Sabbatini F, Chiappa A, Di Cesare S, Lamanna L, et al. Needs of caregivers of the patients with dementia. Arch Gerontol Geriatr. 2010;51(1):54-8.

8. Ryan KA, Weldon A, Huby NM, Persad C, Bhaumik AK, Heidebrink JL, et al. Caregiver support service needs for patients with mild cognitive impairment and Alzheimer disease. Alzheimer Dis Assoc Disord. 2010;24(2):171-6.

9. Jennings LA, Reuben DB, Evertson LC, Serrano KS, Ercoli L, Grill J, et al. Unmet needs of caregivers of individuals referred to a dementia care program. J Am Geriatr Soc. 2015;63(2):282-9.

10. Brodaty H, Thomson C, Thompson C, Fine M. Why caregivers of people with dementia and memory loss don't use services. Int J Geriatr Psychiatry. 2005; 20(6):537-46.

11. Burnell KJ, Selwood A, Sullivan T, Charlesworth GM, Poland F, Orrell M. Involving service users in the development of the support at home: interventions to enhance life in dementia Carer supporter Programme for family carers of people with dementia. Health expect Int J public Particip health care. Health Policy. 2015;18(1):95-110.

12. Novais T, Dauphinot V, Krolak-Salmon P, Mouchoux C. How to explore the needs of informal caregivers of individuals with cognitive impairment in Alzheimer's disease or related diseases? A systematic review of quantitative and qualitative studies. BMC Geriatr. 2017;17(1):86.

13. McCabe M, You E, Tatangelo G. Hearing their voice: a systematic review of dementia family caregivers' needs. The Gerontologist. 2016;56(5):e70-88.

14. Gray SL, Anderson ML, Dublin S, Hanlon JT, Hubbard R, Walker R, et al. Cumulative use of strong anticholinergics and incident dementia: a prospective cohort study. JAMA Intern Med. 2015;175(3):401-7.
15. Carrière I, Fourrier-Reglat A, Dartigues J-F, Rouaud O, Pasquier F, Ritchie K, et al. Drugs with anticholinergic properties, cognitive decline, and dementia in an elderly general population: the 3-city study. Arch Intern Med. 2009;169(14):1317-24.

16. Johnell K. Inappropriate drug use in people with cognitive impairment and dementia: a systematic review. Curr Clin Pharmacol. 2015;10(3):178-84.

17. Thomas $P$, Chantoin-Merlet S, Hazif-Thomas C, Belmin J, Montagne B, Clément $J-P$, et al. Complaints of informal caregivers providing home care for dementia patients: the pixel study. Int J Geriatr Psychiatry. 2002;17(11):1034-47.

18. Thorpe JM, Thorpe CT, Kennelty KA, Gellad WF, Schulz R. The impact of family caregivers on potentially inappropriate medication use in noninstitutionalized older adults with dementia. Am J Geriatr Pharmacother. 2012;10(4):230-41.

19. Wang $X$, Robinson KM, Hardin HK. The impact of caregiving on caregivers' medication adherence and appointment keeping. West J Nurs Res. 2015; 37(12):1548-62.

20. Keeney S, Hasson F, McKenna HP. A critical review of the Delphi technique as a research methodology for nursing. Int J Nurs Stud. 2001;38(2):195-200.

21. Murphy M, Hollinghurst S, Salisbury C. Agreeing the content of a patientreported outcome measure for primary care: a Delphi consensus study. Health expect Int J public Particip health care. Health Policy. 2017;20(2):335-48.

22. Sachdev PS, Blacker D, Blazer DG, Ganguli M, Jeste DV, Paulsen JS, et al. Classifying neurocognitive disorders: the DSM-5 approach. Nat Rev Neurol. 2014;10(11):634-42.

23. Campbell SM, Shield T, Rogers A, Gask L. How do stakeholder groups vary in a Delphi technique about primary mental health care and what factors influence their ratings? Qual Saf Health Care. 2004;13(6):428-34.

24. Revel V, Haritchabalet I, Kervinio C. Construction of a simplified scale for general medicine in detection of caregiver burden of a dependent elderly personAnnée Gérontologique. 2002;16:131-7.

25. Gagnon M, Letenneur L, Dartigues JF, Commenges D, Orgogozo JM, Barberger-Gateau P, et al. Validity of the mini-mental state examination as a screening instrument for cognitive impairment and dementia in French elderly community residents. Neuroepidemiology. 1990;9(3):143-50.

26. Lawton MP, Brody EM. Assessment of older people: self-maintaining and instrumental activities of daily living. The Gerontologist. 1969;9(3):179-86.

27. Cummings $\mathrm{J}$. The neuropsychiatric inventory: assessing psychopathology in dementia patients. Neurology. 1997;48(5 Suppl 6):S10-6.

28. Wackerbarth SB, Johnson MMS. Essential information and support needs of family caregivers. Patient Educ Couns. 2002:47(2):95-100.

29. Carduff E, Finucane A, Kendall M, Jarvis A, Harrison N, Greenacre J, et al. Understanding the barriers to identifying carers of people with advanced illness in primary care: triangulating three data sources. BMC Fam Pract. 2014;15:48.

30. Knighting K, O'Brien MR, Roe B, Gandy R, Lloyd-Williams M, Nolan M, et al. Gaining consensus on family carer needs when caring for someone dying at home to develop the Carers' alert thermometer (CAT): a modified Delphi study. J Adv Nurs. 2016;72(1):227-39.

31. Bond KS, Jorm AF, Kitchener BA, Kelly CM, Chalmers KJ. Development of guidelines for family and non-professional helpers on assisting an older person who is developing cognitive impairment or has dementia: a Delphi expert consensus study. BMC Geriatr. 2016;16:129.

32. Joling KJ, Windle G, Dröes R-M, Huisman M, Hertogh CMPM, Woods RT. What are the essential features of resilience for informal caregivers of people living with dementia? A Delphi consensus examination. Aging Ment Health. 2015:1-9.

33. Dickinson C, Dow J, Gibson G, Hayes L, Robalino S, Robinson L. Psychosocial intervention for carers of people with dementia: what components are most effective and when? A systematic review of systematic reviews. Int Psychogeriatr. 2017;29(1):31-43.

34. Gilhooly KJ, Gilhooly MLM, Sullivan MP, Mclntyre A, Wilson L, Harding E, et al. A meta-review of stress, coping and interventions in dementia and dementia caregiving. BMC Geriatr. 2016;16:106.

35. Sörensen $S$, Pinquart M, Duberstein P. How effective are interventions with caregivers? An updated meta-analysis. The Gerontologist. 2002;42(3):356-72.

36. Novais T, Moutet C, Delphin-Combe F, Dauphinot V, Colin C, Krolak-Salmon P, et al. PHARMAID study protocol: randomized controlled trial to assess the impact of integrated pharmaceutical care at a psychosocial intervention on caregiver's burden in Alzheimer's disease or related diseases. Contemp Clin Trials. 2017;53:137-42.

37. Lindeman CA. Delphi survey of priorities in clinical nursing research. Nurs Res. 1975;24(6):434-41.

38. Ludwig B. Predicting the future: have you considered using the Delphi methodology? J Ext. 1997;35(5):1-4

39. Hearnshaw HM, Harker RM, Cheater FM, Baker RH, Grimshaw GM. Expert consensus on the desirable characteristics of review criteria for improvement of health care quality. Qual Health Care QHC. 2001;10(3):173-8. 